\title{
Cascading Stall of Many Induction Motors in a Simple System
}

\author{
Hao Wu, Member IEEE and Ian Dobson, Fellow IEEE
}

\begin{abstract}
After an initial fault lowers bus voltage, induction motors connected to the bus can successively stall in a cascading process that can lead to a voltage collapse or delayed voltage recovery. There are typically many induction motors connected to the bus, and models that aggregate the motors do not capture the cascading stall. We study the cascading stall in a simple power system with many induction motors using a quasistatic assumption and randomization of some motor parameters, and hence efficiently estimate the distribution of the number of motors stalled. This approach is also extended to a simple 4-bus system and a simple motor dynamics and similar results are obtained. These distributions can often be fit with a high-level and generic probabilistic model of cascading failure, showing a commonality between cascading motor stall and other cascading processes.
\end{abstract}

Index Terms - cascading failure, induction motor, load modeling, voltage stability.

\begin{tabular}{ll} 
& \multicolumn{1}{c}{ NomENCLATURE } \\
$E$ & Voltage magnitude for infinite bus \\
$x$ & Voltage magnitude and angle for load bus \\
$P_{L}, Q_{L}$ & Transmission line reactance \\
$P_{M}, Q_{M}$ & Real and reactive power for total load \\
$M_{\text {ratio }}$ & Real and reactive power for total motor load \\
& Ratio between initial total motor power and \\
$N$ & initial total load power \\
$s$ & Number of motors connected to load bus \\
$k$ & Slip of motor \\
subscript 0 & Load factor of motor \\
subscript $i$ & Indicates quantity at initial state, e.g., $s_{0}$ \\
$V_{c r}, s_{c r}$ & Indicates quantity for motor $i$, e.g., $s_{i}$ \\
$V_{\text {min }}, V_{\text {max }}$ & Critical voltage and slip for motor \\
$P_{r a t i o}$ & Lower and upper limits of distribution of $V_{c r}$ \\
& Ratio of the maximum motor initial power \\
$B_{C i}$ & to the minimum motor initial power \\
$C_{i}$ & Parallel compensator for motor $i$ \\
$Y_{M}$ & Factor converting equivalent admittance for \\
$N_{s}$ & motor $i$ from motor base to system base \\
$P_{0}$ & Admittance of all motors at system base \\
$d, p$ & Number of motors stalled at steady-state \\
& Probability of no motors stalling
\end{tabular}

\section{INTRODUCTION}

Induction motors are a significant dynamic load in power systems. They typically consume about $57 \%$ of total load

Hao $\mathrm{Wu}$ is with the College of Electrical Engineering, Zhejiang University, Hangzhou, Zhejiang, China, 310027.vuhao@zju.edu.cn. Ian Dobson is with the Electrical and Computer Engineering Department, Iowa State University, Ames IA 50011 USA. dobson@iastate.edu. Financial support in part from China NSF grant 50707029 (Wu), and USA DOE grant DESC0002283 and NSF grant CPS-1135825 (Dobson) is gratefully acknowledged. CIEEE 2012 power and about $78 \%$ of industry loads [1]. Hence their behavior has crucial impacts on power system dynamic behavior [2], especially, voltage stability and voltage performance [1], [3], [4], [5]. Because of this, modeling induction motors has attracted researchers over the last thirty years.

The early works about induction motor modeling are well summarized in [6], [7], where the induction motors at one substation are aggregated into one equivalent induction motor. Combined with some parallel static load components, such as a constant impedance load, it forms the substation load model that is both simple and commonly used.

However, some operational problems have stimulated research in more elaborate models. The operational problems include fast voltage instability and power oscillation problems in bulk power systems [8], [9] and fault-induced delayed voltage recovery problem at several voltage levels [5], [10], [11].

Because induction motor cascading stall can lead to fast voltage instability, [12], [13] and [14] studied this mechanism based on numerical simulation and bifurcation theory. Two or more motors are modeled there, but each load bus has only one aggregate motor. The studies of the 1996 WSCC system oscillations stress the importance of dynamic load model parameters [9], [15]. This motivated research on the influence of load model parameter uncertainties on system eigenvalues [16], [17] and the characteristic and clustering of load modeling at one substation [18]. However, in these studies, each load bus still has one aggregate motor. The studies of delayed voltage recovery are interesting. Besides cascading stall and parameter variability problems, they require incorporating air conditioner compressor models along with their protection logic into the load model [5], [10], [19], [20]. Hence the resulting load models include several equivalent motors, one of which can stall more easily. In summary, we note the trends in recent studies of induction motor models of considering parameter uncertainties and more equivalent motors and the problem of cascading stall.

As regards the general phenomenon of cascading failure, bulk power transmission systems experience cascading events which can cause large blackouts [21], [22]. There has been recent progress in describing both simulated and observed cascading failures in transmission systems using high-level probabilistic models, such as the loading-dependent model CASCADE [23] or the branching process models that approximate CASCADE [24], [25], [26], [27]. As well as providing insights into the cascading process, CASCADE provides a simple formula that predicts the number of failed elements in terms of parameters that describe the size of the initial disturbance and the average amount of propagation of failures. In this paper we show that the CASCADE formula can also fit the cascading stall of induction motors. 


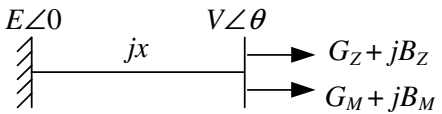

Fig. 1. Single load infinite bus system

Considering the power system reality that there can be thousands, even millions of induction motors connected through the distribution system to one substation and the motors vary in type and operating conditions, the uncertainty in motor parameters and operating conditions can be substantial. Therefore, their critical stability conditions are dispersed, which implies that there is a range of propensities for motor stall. Hence it is appropriate to model the variability and uncertainty of the motors probabilistically. In particular, we model the critical stall voltage and the motor size probabilistically.

While the load model with one or a few equivalent induction motors can be very useful for many purposes, the cascading stall of many motors cannot be studied through this model. Instead, we assume many induction motors and for illustrative purposes use 100 motors. Because so many motors will be simulated and it is a first analysis in this style, it is appropriate to use the simplest motor models that can capture the cascading stall. In particular, we use a quasistatic assumption to capture the main features of the cascading motor stall and avoid the detailed simulation of the motor dynamics.

We study the voltage collapse when induction motors successively stall after an initial disturbance that depresses the bus voltage. Since each motor stall tends to draw more current and further depress the voltage, there can be a cascade of motors stalling. The cascade is one typical mechanism leading to voltage instability or fault-induced delayed voltage recovery. This paper describes a quasistatic model that summarizes the cascading process of voltage decline and then shows that the number of motors stalled can often be fit with the CASCADE model [23].

There is related initial work in [28] that, in contrast the the present paper, assumes a uniform distribution of initial slip and equal initial motor powers. This paper treats different cases and extends the general direction of modeling in [28] by describing further insights into the results and their relation to the CASCADE model.

The organization of the paper is as following. Section II describes the power system and induction motor modeling. The quasistatic model for the cascading stall is proposed in section III. Section IV reviews the CASCADE model. Section $\mathrm{V}$ presents the simulation results and their fit with the CASCADE model. Section VI suggests a fast analysis method that can efficiently derive the contours of equal cascading probability in a parameter plane. Section VII discusses how the approach could apply to multi-bus systems, and suggests future research directions. Section VIII shows a simple dynamic case study and section IX compares our probabilistic approach with a composite dynamic load model approach. Section X concludes the paper.

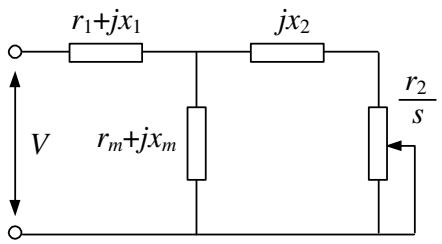

Fig. 2. T-shaped equivalent circuit for induction motor

\section{Modeling AND ASSUMPTIONS}

\section{A. Power system model}

For the single load infinite bus system shown in Fig. 1, the power flow equations are

$$
\begin{aligned}
-\frac{E V}{x} \sin \theta & =P_{L}=\left(G_{Z}+G_{M}\right) V^{2} \\
\frac{E V}{x} \cos \theta-\frac{V^{2}}{x} & =Q_{L}=-\left(B_{Z}+B_{M}\right) V^{2}
\end{aligned}
$$

where $G_{Z}+j B_{Z}=Y_{Z}$ is a constant parallel load admittance, $G_{M}+j B_{M}=Y_{M}$ is an equivalent admittance for the total motor load, and $P_{L}+j Q_{L}$ is total load power. The ratio between initial total motor power and initial total load power is $M_{\text {ratio }}$ and the total motor power is $P_{M}+j Q_{M}$. Solving (1) for the bus voltage gives

$$
V=\frac{E}{x \sqrt{\left(G_{Z}+G_{M}\right)^{2}+\left(B_{Z}+B_{M}-\frac{1}{x}\right)^{2}}}
$$

\section{B. Induction motor model}

Assume there are $N$ induction motors attached in parallel to the load bus. Each induction motor is modeled by the well known T-shaped equivalent circuit shown in Fig. 2, where $s$ is motor slip, and $r_{1}, x_{1}, r_{2}, x_{2}, r_{m}$ and $x_{m}$ are resistances and inductances for the stator, rotor and magnetic windings respectively. The electromagnetic torque $T_{e}$ is

$$
T_{e}(s, V)=\frac{r_{2}}{s}\left|\frac{z_{m}}{z_{1} z_{m}+\left(z_{m}+z_{1}\right) z_{2}(s)}\right|^{2} V^{2},
$$

where $z_{1}=r_{1}+j x_{1}, z_{m}=r_{m}+j x_{m}$, and $z_{2}(s)=r_{2} / s+j x_{2}$. The mechanical torque $T_{m}$ is

$$
T_{m}(s)=k\left[\alpha+(1-\alpha)(1-s)^{p}\right]
$$

where $k$ is load factor, $\alpha$ is the ratio of constant mechanical torque to total torque, and $p$ is a factor reflecting the impact of motor rotation speed on the non-constant mechanical torque component. The rotor dynamic equation is

$$
T_{J} \frac{d s}{d t}=T_{m}(s)-T_{e}(s, V),
$$

where $T_{J}$ is total inertia constant for the motor rotor and its mechanical load.

\section{Simplifying assumptions and critical voltage $V_{c r}$}

A detailed simulation including the induction motor slip dynamics based on (5) can be useful, especially for a small number of induction motors [12], [13], [14]. However, for 


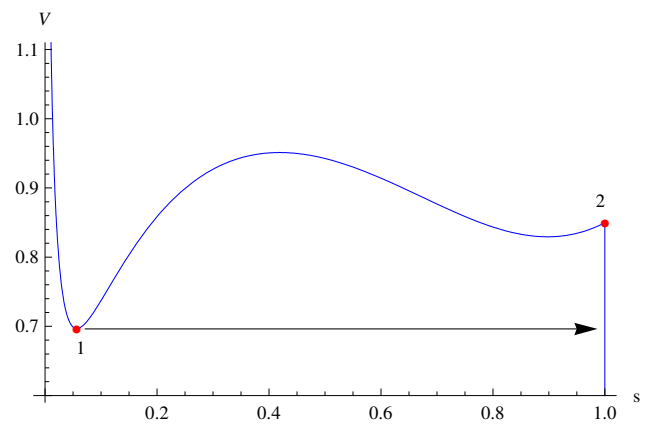

Fig. 3. Relationship between voltage $V$ and slip $s$ for a single motor.

hundreds of induction motors, such a detailed dynamical simulation is time-consuming and even intractable if Monte Carlo simulation is used. Therefore we model the cascading stall of the system quasistatically as a series of steady states. The motors that are stalled are modeled by the constant admittance

$$
Y_{M T}^{s}=\frac{z_{2}+z_{m}}{z_{1} z_{2}+z_{1} z_{m}+z_{2} z_{m}}
$$

where $z_{2}=r_{2}+j x_{2}$, while the motors that are not stalled satisfy the static torque balance equation

$$
T_{m}(s)=T_{e}(s, V) .
$$

All variables in (7), except $V, s$, and $k$, are motor parameters. Because $k$ is determined when the motor initializes and never changes later, (7) describes the relationship between $V$ and $s$ for a single motor, as shown in Fig. 3. The stalled state corresponds to slip $s=1$ and the line below point 2 in Fig. 3. The normal stable operation range, where $s$ is small, corresponds to the portion of Fig. 3 to the left of point 1 . As the rest of system changes by small amounts, the motor voltage will decrease quasistatically along the portion of Fig. 3 to the left of point 1 until it reaches the critical voltage $V_{c r}$ and critical slip $s_{c r}$ at the local minimum of the curve, at which point the steady state solution is lost and the motor stalls with $s=1$ as indicated by the arrow. ${ }^{1}$ A similar quasistatic assumption has been very useful in bifurcation analysis of motor dynamics [13]. This modeling is consistent with the motor voltage always decreasing as the initial fault occurs and as the other motors stall. In this paper we focus on the voltage decline caused by cascading stall, and do not consider any process of voltage recovery as motors resume normal operation, or as motors or other loads are disconnected by protection.

We assume that all motors have same parameters as given in the appendix, except for $V_{c r}, k$ and the initial slip $s_{0}$. To

\footnotetext{
${ }^{1}$ The portion of the curve in Fig. 3 to the right of point 1 can, depending on machine parameters, include some states intermediate between normal operation and stall that could be potentially accessible when certain motor transients are considered. Neglecting the existence of these states will not cause much problem, because these states requires a relatively high $V$ and, even if they are encountered, the motor draws a large current similar to that drawn by a motor stalled with $s=1$.
}

determine the critical slip $s_{c r}$, rewrite (7) as

where

$$
\frac{V^{2}}{k}=h(s) \text {, }
$$

$$
h(s)=\left[\alpha+(1-\alpha)(1-s)^{p}\right] \frac{s}{r_{2}}\left|z_{1}+\left(1+\frac{z_{1}}{z_{m}}\right) z_{2}(s)\right|^{2} .
$$

Since $V_{c r}$ is a local minimum at $s=s_{c r}, h(s)$ also has a local minimum at $s=s_{c r}$, and hence it is straightforward to calculate $s_{c r}$. Since all variables in $h(s)$ except $s$ are parameters common to all the motors, $s_{c r}$ is the same for all the motors.

The critical voltage $V_{c r}$ will vary among the motors, and we make the simple assumption that $V_{c r}$ is a random variable uniformly distributed in an interval $\left[V_{\min }, V_{\max }\right]$.

Although total initial motor power is fixed, the initial power $P_{M 0 i}$ of motor $i$ is chosen randomly. Let the ratio of the maximum motor initial power to the minimum motor initial power be not greater than $P_{\text {ratio }}$. We assume that the weight of each motor's initial power $P_{M 0 i}$ in total motor initial power $P_{M 0}$ is a uniform random variable in the interval $\left[1, P_{\text {ratio }}\right]$. Then, $P_{M i}$ can be allocated from $P_{M 0}$ according to its weight.

\section{Induction motor initialization}

Now we initialize the remaining parameters for the $i$ th induction motor for $i=1,2, \ldots, N$. The critical voltage $V_{c r i}$ is sampled from the uniform distribution in the interval $\left[V_{\min }, V_{\max }\right]$. Then $k_{i}$ is calculated using (8):

$$
k_{i}=\frac{V_{c r i}^{2}}{h\left(s_{c r}\right)},
$$

and the motor initial slip $s_{0 i}$ is obtained from (7):

$$
s_{0 i}=S_{0}\left(V_{c r i}\right) .
$$

Since $V_{c r i}$ has an uniform distribution, $s_{0 i}$ will not be uniformly distributed due to the nonlinear relationship (10). One consequence is that initially there will tend to be fewer motors running at high slip.

From the T-shaped equivalent circuit in Fig. 2, the equivalent admittance $Y_{M T i}$ for motor $i$ is

$$
Y_{M T i}\left(s_{i}\right)=\frac{z_{m}+z_{2}\left(s_{i}\right)}{z_{1} z_{m}+\left(z_{1}+z_{m}\right) z_{2}\left(s_{i}\right)}
$$

Motor $i$ has a parallel compensator with susceptance $B_{c i}$ attached to the motor terminal to make the compensated motor power factor equal to the power factor of the initial total bus load $P_{L 0}+j Q_{L 0}$. The value of $B_{c i}$ is determined during the initialization by

$$
B_{c i}=-\operatorname{Re}\left(Y_{M T i}\left(s_{0 i}\right)\right) \frac{Q_{L 0}}{P_{L 0}}-\operatorname{Im}\left(Y_{M T i}\left(s_{0 i}\right)\right)
$$

Once $B_{c i}$ is determined, it never changes, and the motor remains compensated when it stalls. Therefore the total admittance of motor $i$, including the compensation, is

$$
Y_{M C i}= \begin{cases}Y_{M C i}^{n}=Y_{M T i}\left(s_{i}\right)+j B_{c i} & \text { if } V_{c r i} \leq V, \\ Y_{M C i}^{s}=Y_{M T}^{s}+j B_{c i} & \text { if } V_{c r i}>V .\end{cases}
$$

The above motor admittances are in the motor base and need 
to be converted to the system base. It is a common practice in induction motor simulation that the admittance ratio between system base and motor base is assumed to be a constant $C_{i}$ at any moment during the dynamic process [29]; that is,

$$
C_{i}=\frac{Y_{M i}}{Y_{M C i}},
$$

where $Y_{M i}$ is equivalent admittance of motor $i$ in system base. Since all variables at initial state can be obtained, $C_{i}$ can be determined. Let motor $i$ initial equivalent admittance in system base is $Y_{M 0 i}$. Then

$$
C_{i}=C\left(S_{0}\left(V_{c r i}\right)\right)=\frac{Y_{M 0 i}}{Y_{M C i}\left(s_{0 i}\right)}=\frac{P_{M 0 i}-j Q_{M 0 i}}{Y_{M C i}\left(s_{0 i}\right) V_{0}^{2}} .
$$

\section{E. Induction motor admittance}

From (8) we know that $s_{i}$ is a function of $V$ and $k_{i}$, and hence, a function of $V$ and $V_{c r i}$ because of (9). Thus, from (13) and (14) we have the compensated motor $i$ admittance in system base:

$$
Y_{M i}= \begin{cases}Y_{M i}^{n}\left(V, V_{c r i}\right)=C_{i} Y_{M C i}^{n} & \text { if } V_{c r i} \leq V \\ Y_{M i}^{s}\left(V_{c r i}\right)=C_{i} Y_{M C i}^{s} & \text { if } V_{c r i}>V .\end{cases}
$$

The equivalent admittance $Y_{M}$ of the total motor load is

$$
Y_{M}(V)=\sum_{i=1}^{N} Y_{M i} .
$$

\section{QuasistaTiC MODEL OF CASCADING STALL}

After the initial fault on the power system, the cascading motor stall proceeds as follows: As the bus voltage $V$ drops, the motor with the highest critical voltage $V_{c r}$ will stall first. The stalled motor will be modeled by (6) and draw more current from the system. This causes the bus voltage $V$ to drop further. If $V$ falls below $V_{c r}$ of some remaining unstalled motor, then that motor will also stall. The process continues in a similar way until no further motors stall. Given the induction motor modeling explained above, we can now model the cascading stall of many motors in stages. The objective is to compute the distribution of the number of motors stalled.

Suppose at some stage of cascading, we know which motors are stalled. Then we can compute the resulting bus voltage as follows: Substituting $G_{M}+j B_{M}$ in (2) by $Y_{M}(V)$ in (17) will form a fairly complicated equation for $V$. The solution of the complicated equation is the post-fault steady state voltage $V$, which is denoted as $V_{s}$ hereafter. The solution $V_{s}$ is obtained iteratively.

The quasistatic model uses random motor critical voltages and powers, and must be run $N_{\text {sample }}$ times to sample these random variables and produce $N_{\text {sample }}$ samples of the number of motors stalled. Denote the pre-fault and post-fault transmission line reactance as $x_{n}$ and $x_{p}$ respectively. The algorithm may be summarized:

1) Solve $V_{0}$ from (1) with $x=x_{n}, P_{L}+j Q_{L}=P_{L 0}+$ $j Q_{L 0}$, then calculate $G_{Z}+j B_{Z}$.

2) Set sample counter $n=1$.

3) Sample $V_{c r i}$ and weight of initial power for each motor.

4) Allocate total initial motor power to each motor.
5) Initialize each motor to get $k_{i}, B_{c i}$ and $C_{i}$.

6) Give an estimate for bus voltage $V_{s}$.

7) Update each motor's slip $s_{i}$ according to $V_{s}$. More specifically, motors with $V_{c r i}>V_{s}$ have $s_{i}=1.0$, while for those with $V_{c r i} \leq V_{s}$ update $s_{i}$ according to (7).

8) Calculate $Y_{M}$ according to (17) and then solve $V$ from (2) with $G_{M}+j B_{M}=Y_{M}$ and $x=x_{p}$.

9) If the error between $V$ and $V_{s}$ is small enough, goto 10 ; otherwise, construct a new estimate for $V_{s}$ and then goto 7 .

10) If $n<N_{\text {sample }}$, set $n:=n+1$ and goto 3. Otherwise, output distribution of number of stalled motors and stop.

If the assumptions made in section II-C are satisfied, this simulation algorithm can be more than 1000 times faster than strict time domain simulation based on dynamic equations (5) and at the same time without losing accuracy.

Moreover, above quasistatic simulation algorithm can be easily extended to a multi-bus system, where motors at each bus are processed in the similar way and $V$ for each bus is updated through solving power flow.

\section{CASCADE MODEL}

The CASCADE model is an analytically tractable probabilistic model of cascading failure that captures the weakening of the system as the cascade process [23]. There are $N$ identical components and each component has a level of loading or stress. The normalized initial load on each component is uniformly distributed between zero and one and independent of the initial loading on other components. (Here it is convenient to use the version of CASCADE with all parameters normalized.) There is a normalized initial disturbance $d$ that adds additional loading to every component. Each component fails if its loading exceeds one. When any component fails, all the other components are additionally loaded with a load increment $p$. Initial failures can lead to a cascading sequence of failures as components successively overload and additionally load the other components. The cascade continues until there are no further failures or all the components are failed.

The parameter $d$ is proportional to the size of the initial disturbance, and the parameter $p$ is proportional to how much the cascades propagate. There is no cascading for $d<0$, and nontrivial cascades become possible for $d \geq 0$. For $d \geq 0$, the total number of failed components has the probability distribution:

$$
\begin{aligned}
& \text { Probability of } r \text { total failures }= \\
& \begin{cases}\left(\begin{array}{c}
N \\
r
\end{array}\right) d(r p+d)^{r-1}(\max \{1-r p-d, 0\})^{N-r} ; & r<N, \\
1-\sum_{s=0}^{N-1} \text { probability of } s \text { total failures; } & r=N .\end{cases}
\end{aligned}
$$

In particular, the probability of zero motors stalling is $P_{0}=$ $(1-d)^{N}$.

The classic least squares method is used to fit the distribution of the number of motors stalled from the quasistatic model with the CASCADE model distribution (18). Because the probabilities of numbers of motors stalled can differ by as much as a factor of $10^{4}$, fitting without preprocessing will tend 


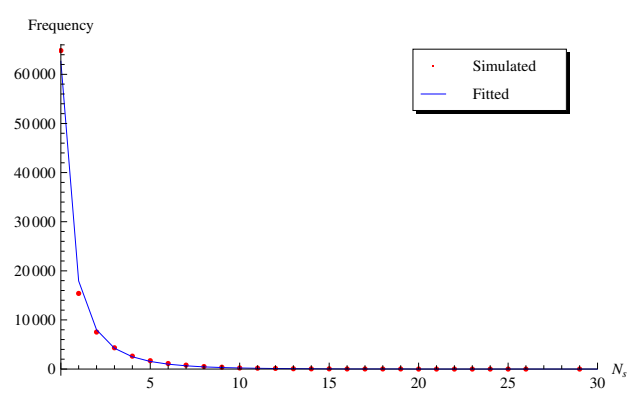

(a) Frequency vs. $N_{s}$.

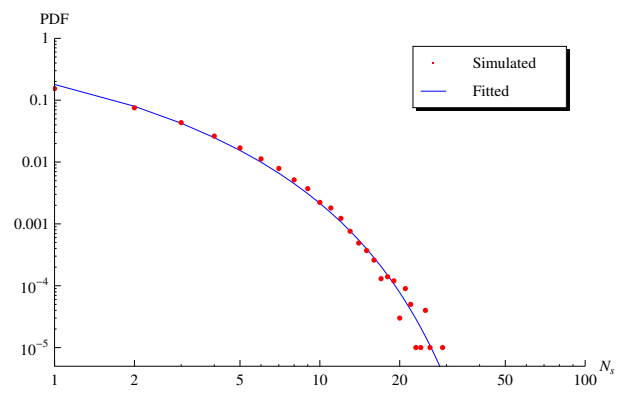

(b) Probability vs. $N_{s}(\log -\log )$

Fig. 4. Distribution of number of motors stalled $N_{s}$ at base case.

to neglect low probability cases. In order to solve this problem, the logarithm of (18) is fit to the logarithm of probabilities.

\section{QUASISTATIC MODEL RESUlTS \& FIT WITH CASCADE}

\section{A. Base case}

The power system parameters are $E=1.029, x_{n}=0.1$ and $P_{L 0}+j Q_{L 0}=1.0+j 0.4$. With these settings, $V_{0}=$ 0.9833. There are $N=100$ motors, with the parameters in the Appendix. Assume $V_{\max }=0.92$ and $V_{\min }=0.5$; that is, some motors begin to stall when $V<0.92$ and all of them stall when $V<0.5$. Assume $P_{\text {ratio }}=10$ and let $M_{\text {ratio }}=0.6$ and $x_{p}=0.2$. The number of samples $N_{\text {sample }}=10^{5}$.

Fig. 4 shows the base case distribution of the total number of failures, where Fig. 4a and Fig. 4b give the frequency and probability distribution of $N_{s}$ respectively. The dots in the figure correspond to simulation result, while the curve shows the fitting result from the CASCADE model. Fig. 4a gives the same data as Fig. 4b, but the log-log scale in Fig. 4b allows the small probabilities of many motors stalled in the tail of the distribution to be seen. These small probability events are important because many motor stalled have a high impact when they cause a blackout.

\section{B. Impact of load level}

We examine the effect of changing the initial load power $P_{L 0}+j Q_{L 0}$ by scaling it by a factor $\lambda$ while leaving the other parameters unchanged. $\lambda$ is varied between 0.96 and 1.25.

$P_{0}$ is the probability of no motors stalling. In the base case, $P_{0}=0.65$; that is, $35 \%$ of the samples have motors cascading. $P_{0}$ decreasing below one gives the onset of cascading stall. As shown by the fitted line in the semilog plot shown in fig. 5, $P_{0}$ decreases approximately exponentially as the load increases

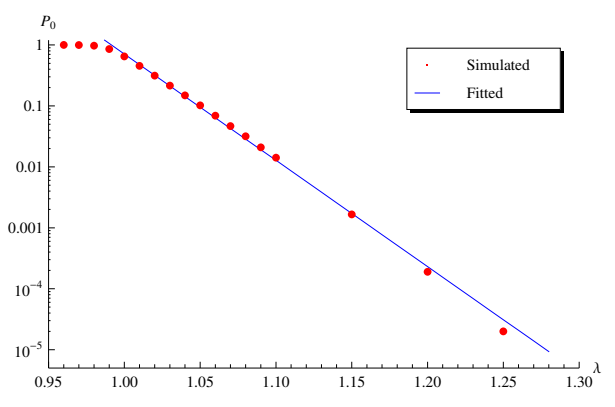

Fig. 5. Probability of no stall $P_{0}$ vs. loading factor $\lambda$

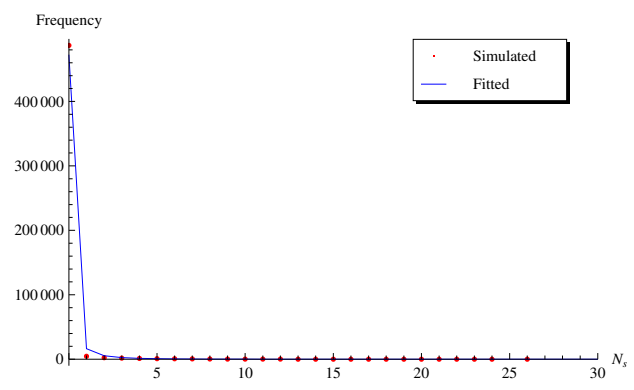

(a) Frequency vs. $N_{s}$.

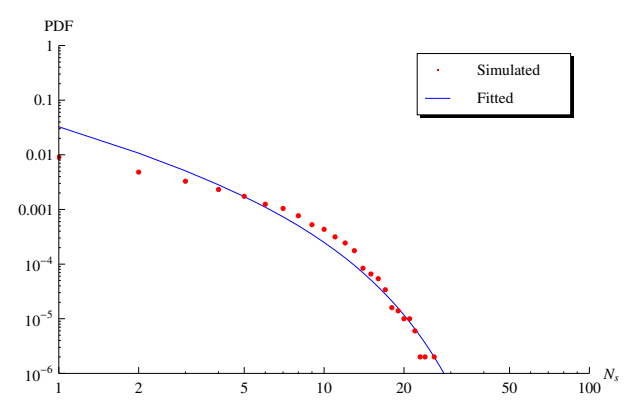

(b) Probability vs. $N_{s}(\log -\log )$

Fig. 6. Distribution of number of motors stalled $N_{s}$ at $\lambda=0.98$

beyond the onset of cascading stall. This shows that a small changes in parameters, say a $5 \%$ increase in load, can strongly affect the probability of cascading.

Figs. 6 to 8 give the distribution of the number of motors stalled when the load factor $\lambda$ is $0.98,1.07$, and 1.25 respectively. As we can see from these figures and Fig. 4, when $\lambda$ increases, and the system is more stressed, the chance of more motors stalled increases. The distribution in Fig. 8 for $\lambda=1.25$ is approximately normal. All plots above show that the CASCADE model can fit the results quite well.

The base case $\lambda=1$ is likely to be a cascading case of engineering interest since it is intermediate between the case of lower stress $\lambda=0.98$ in which cascades are small and cascading is not fully developed, and the highly stressed cases $\lambda=1.07$ or 1.25 , in which cascading is severe and many motors stalled is very likely.

The above impact of $\lambda$ on the distribution of the number of motors stalled is quite typical. Similar patterns can be observed if $P_{L 0}, Q_{L 0}$ or $M_{\text {ratio }}$ changes around the base case. Due to space limitations, they are not described here.

We have varied the maximum ratio of initial motor powers 


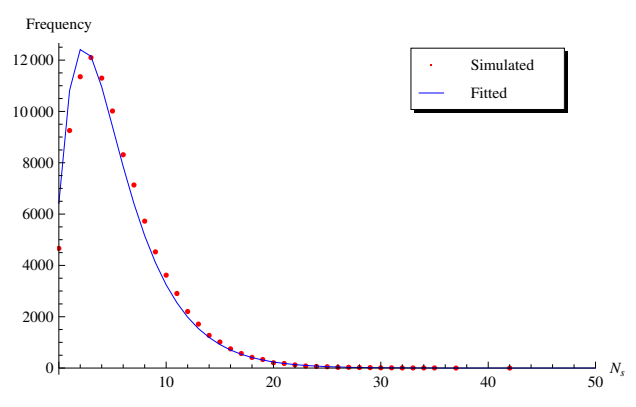

(a) Frequency vs. $N_{s}$.

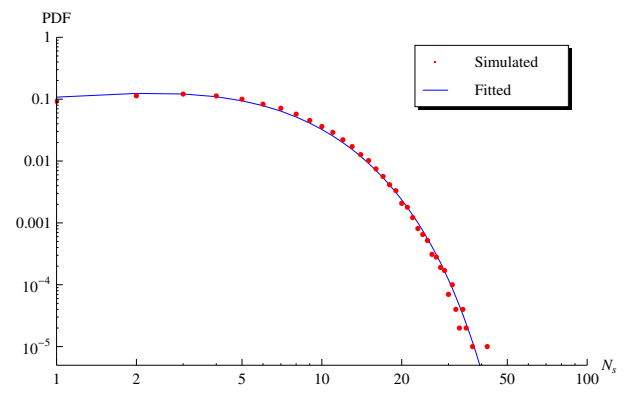

(b) Probability vs. $N_{s}(\log -\log )$

Fig. 7. Distribution of number of motors stalled $N_{s}$ at $\lambda=1.07$

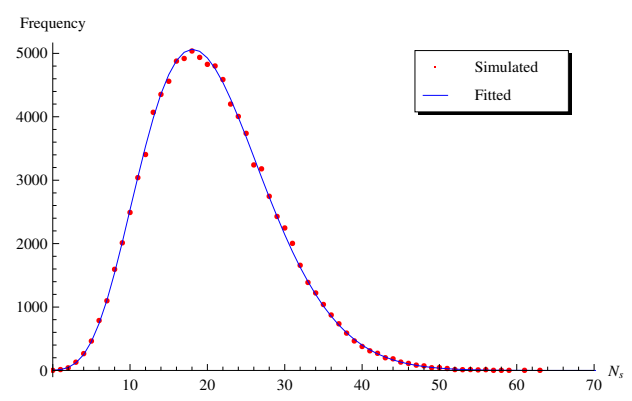

(a) Frequency vs. $N_{s}$.

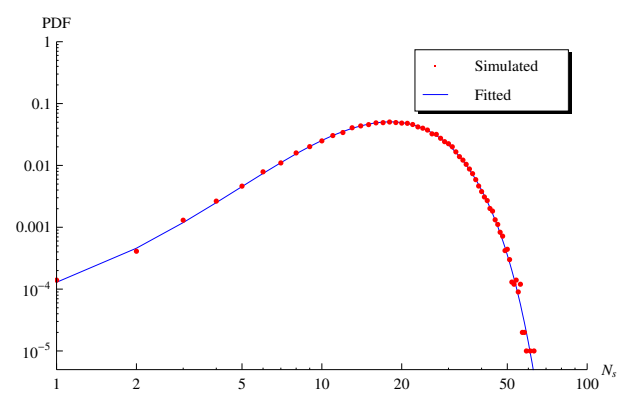

(b) Probability vs. $N_{s}(\log -\log )$

Fig. 8. Distribution of number of motors stalled $N_{s}$ at $\lambda=1.25$

from $P_{\text {ratio }}=1$ (all motors with the same initial power) to $P_{\text {ratio }}=100$ and found that the distribution of the number of motors stalled is insensitive to $P_{\text {ratio }}$. We conclude that for this calculation it is a good approximation to assume all motor powers equal.

\section{Highly stressed cases}

Fig. 9 shows the match of the distribution of $N_{s}$ with CASCADE in 3 cases with load powers $0.96+j 0.384,1.188+j 0.3$,

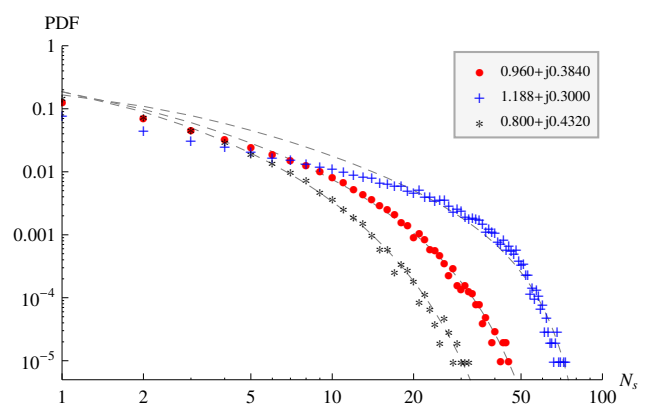

Fig. 9. Distribution of number of motors stalled $N_{s}$ for three cases when $M_{\text {ratio }}=0.8$

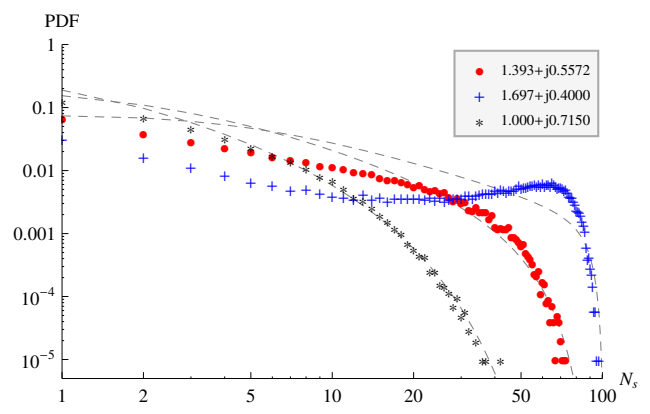

Fig. 10. Distribution of number of motors stalled $N_{s}$ for three cases when $V_{\max }=0.85$.

and $0.8+j 0.432$ respectively, when $M_{\text {ratio }}=0.8$. The corresponding $P_{0}$ are $0.5950,0.5933$, and 0.6184 , all still about 0.6. In terms of the CASCADE model, the higher stress is indicated by $d+N p=d+100 p$ getting bigger. $d+N p$ of second case is estimated to be 0.7738 , which is larger than 0.6474 of first case and 0.5358 of third case. The system is highly stressed with significant probability of large numbers of motors stalled. The distribution of $N_{s}$ in the second case is harder to fit.

Fig. 10 compares the distribution of $N_{s}$ for three cases when $V_{\max }=0.85$. Their load powers are $1.393+j 0.5572,1.697+$ $j 0.4$ and $1.0+j 0.715$ respectively. Their $P_{0}$ are $0.5990,0.5644$ and 0.6159 , still about 0.6 . With these parameters, $d+N p$ for the three cases are estimated to be $0.7870,0.9119$ and 0.6006 respectively. Because the second case has a high probability of stalling many motors, its $d+N p$ is close to 1 and the distribution is badly fitted. Because the third case has a small $d+N p$, it still can be well fitted. The fitting result of the first case is marginal. We conclude that for some highly stressed cases, the distribution of $N_{s}$ can not be well fitted with the CASCADE model.

\section{Distribution of other variables after cascading}

The number of motors stalled is a convenient measure of the size of the cascade, but other variables such as the final values of steady-state load bus voltage $V_{s}$, angle $\theta_{s}$, load power $P_{L s}$ and $Q_{L s}$ may be easier to measure in practice. Therefore we examine the distributions of these variables.

The final values of $V_{s}$ and $P_{L s}$ decrease as the number of motors stalled increases. To make all variables increase as the number of motors stalled increases, we consider $\operatorname{Max}\left(V_{s}^{n}\right)-$ 


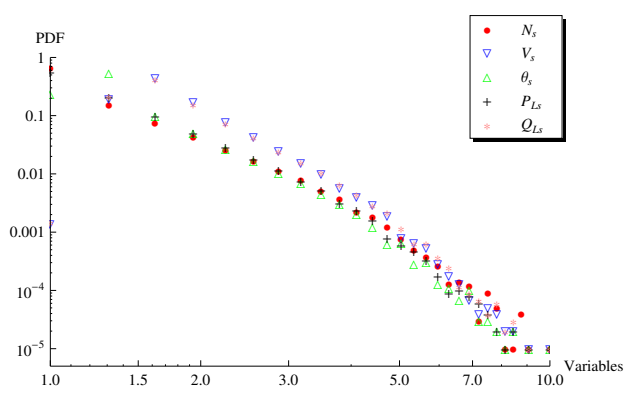

Fig. 11. Distribution of multiple variables after cascading at base case.

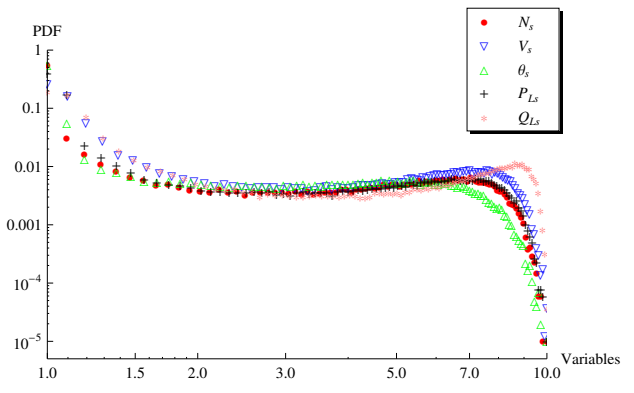

Fig. 12. Distribution of multiple variables after cascading when $V_{\max }=$ 0.85 and $P_{L 0}+j Q_{L 0}=1.697+j 0.4$.

$V_{s}^{n}$ and $\operatorname{Max}\left(P_{L s}^{n}\right)-P_{L s}^{n}$, where superscript $n$ stands for the $n$th sample point of corresponding variable in its distribution range. To be able to compare the distributions, we normalize the range of $N_{s}^{n}, \operatorname{Max}\left(V_{s}^{n}\right)-V_{s}^{n}, \theta_{s}^{n}, \operatorname{Max}\left(P_{L s}^{n}\right)-P_{L s}^{n}$ and $Q_{L s}^{n}$ to the range 1 to 10 and plot all five distributions for the base case in Fig. 11. All five distributions have a similar form in the base case. This conclusion also holds in the more stressed system in which $V_{\max }=0.85$ and $P_{L 0}+j Q_{L 0}=$ $1.697+j 0.4$ as shown in Fig. 12 .

\section{A TYPICAL SAMPLE AND CONTOURS}

The quasistatic model is significantly faster than full dynamic simulation, but it requires many samples of the motor critical voltages and initial powers, which is still quite time consuming. For the quickest analysis, the quasistatic model can be used on one typical sample of the motor critical voltages and initial powers. The typical sample of the critical voltages is taken as the equally spaced sample

$$
V_{c r i}=V_{\min }+\left(V_{\max }-V_{\min }\right) \frac{i-0.5}{N}, \quad i=1,2, \ldots, N \text {. }
$$

The motor initial powers are simply assumed to be all equal; i.e., $P_{M 0 i}+j Q_{M 0 i}=\left(P_{M 0}+j Q_{M 0}\right) / N$. With this typical sample, the quasistatic model then gives a typical number $\tilde{N}_{s}$ of motors stalled. This fast computation allows combinations of parameters that give the same number $\tilde{N}_{s}$ of typical stalled motors to be obtained and plotted as a contour as shown in Fig. 13.

Fig. 13 shows three contours in $P_{L 0}-Q_{L 0}$ plane with $\tilde{N}_{s}$ equal to 0,5 , and 10 respectively. All parameters other than $P_{L 0}$ and $Q_{L 0}$ are the same as base case. The contour for $\tilde{N}_{s}=$

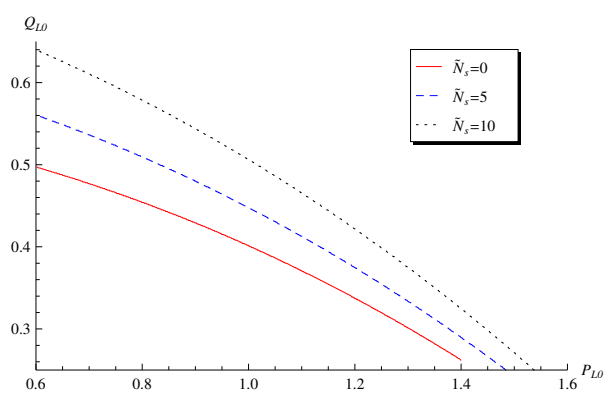

Fig. 13. Contours of constant $\tilde{N}_{s}$ (typical number of motors stalled) as $P_{L 0}$ and $Q_{L 0}$ vary from the base case.

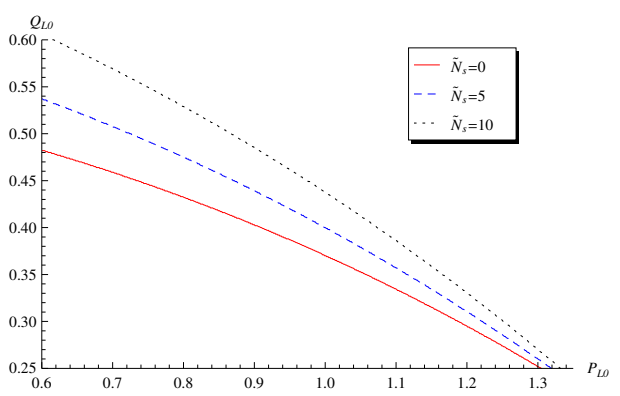

Fig. 14. Contours of constant $\tilde{N}_{s}$ as $P_{L 0}$ and $Q_{L 0}$ vary when $M_{\text {ratio }}=0.8$

0 corresponds to the onset of cascading in the typical sample, This $\tilde{N}_{s}=0$ contour is related to, but not the same as, the onset of cascading in the quasistatic model or CASCADE. The onset of cascading in the quasistatic model corresponds to the voltage following the initial disturbance falling to the highest $V_{c r}$. Similarly, the onset of cascading in the CASCADE model is $d$ becoming positive. In the typical sample, the highest motor critical voltage is $V_{\max }-\frac{1}{2 N}\left(V_{\max }-V_{\min }\right)$, which in the base case is 0.9179 . The cascades start when $d=\frac{V_{\max }-0.9179}{V_{\max }-V_{\min }}=$ 0.005 . When $d=0.005, P_{0}=(1-d)^{100}=0.606$. Therefore the $\tilde{N}_{s}=0$ contour corresponds to $P_{0}=0.606$.

The closer spacing of the contours in Fig. 13 for higher real power load and lower reactive load indicates that the cascading process progresses from no stall to many motors stalled more quickly when the real load is increased. For example, this applies when reactive power compensation to the load increases the real power that the load can consume.

Increasing $M_{\text {ratio }}$ to 0.8 and holding the other parameters unchanged, the contours change to Fig. 14. Compared with Fig. 13, the area inside inner contour is shrunk and the contours are much closer at high $P_{L 0}$. This suggests that the system is more stressed.

Fig. 15 gives the contours when $V_{\max }=0.85$ while the other parameters remain unchanged. Lower $V_{\max }$ means the motors are hard to stall, and hence the area inside inner contour is enlarged a lot compared with Fig. 13. But lower $V_{\max }$ also means all $V_{c r i}$ are distributed more closely, which implies that once motors start to stall it is easier for many of them to stall. Therefore, the contours in Fig. 15 are very close. In the region of large $P_{L 0}$, the contours of $\tilde{N}_{s}$ equaling to 5 and 10 are missing, which means that for the typical sample, more than 10 motors will stall if the stalling starts. 


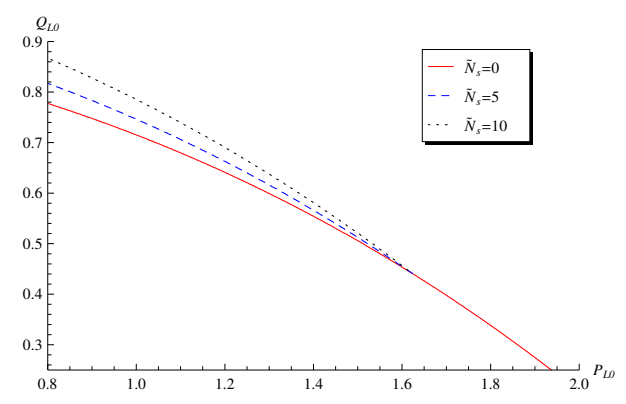

Fig. 15. Contours of constant $\tilde{N}_{s}$ as $P_{L 0}$ and $Q_{L 0}$ vary when $V_{\max }=0.85$

From above case studies, it can be seen that the information about $\tilde{N}_{s}$ is quite useful. The transition of $\tilde{N}_{s}$ from 0 to 1 roughly corresponds to $P_{0} \approx 0.6$. Moreover, the distance between different $\tilde{N}_{s}$ contours can be used to indicate the sensitivity of the cascading process to parameters. If this distance is large; i.e., $\tilde{N}_{s}$ changes slowly when parameters vary, then the range of the likely number of motors stalled is smaller and can be well fitted to CASCADE model. If this distance is small so that $\tilde{N}_{s}$ changes abruptly when parameters vary, then the range of the likely number of motors stalled is larger and is harder to fit with the CASCADE model. From an engineering viewpoint, the former situation is preferred because the latter implies a sudden onset of massive cascading stall.

\section{EXTENSION TO MULTI-BUS SYSTEMS}

This section discusses how methods for single load systems could be enhanced and then applied to multibus systems. The previous sections show the possibility of characterizing the cascading risk due to the stall of many induction motors with a stochastic cascading failure model with two parameters $d$ and $p$ that describe the size of the initial disturbance and its tendency to propagate.

One challenge is to understand how $d$ and $p$ can be derived from measurements at a load bus, and how they can be influenced by system operational changes such limits on the power flows or design changes such as adding reactive power support to the load bus. The measurements would be taken for the more likely and frequent smaller cascades, and the purpose would be to quantify the cascading risk at the bus so that the infrequent but high impact large cascades could be mitigated. It is necessary to base the approach on measurements of bulk behavior at the load bus, because it is not feasible to get data for or accurately model hundreds or thousands of induction motors attached to a feeder. Although this paper initially illustrates some qualitative effects of power flows or bus parameters on $d$ and $p$, this challenge will be fully addressed in a companion paper that includes analytic approximations for $d$ and $p$.

Given measurements of $d$ and $p$ at vulnerable load buses, one could predict the probability of cascading failure at that bus by computing with the CASCADE model the probability distribution of the proportion of motor load stalled. One simple initial approach is that mitigation efforts could proceed separately at each bus, starting with the most vulnerable load

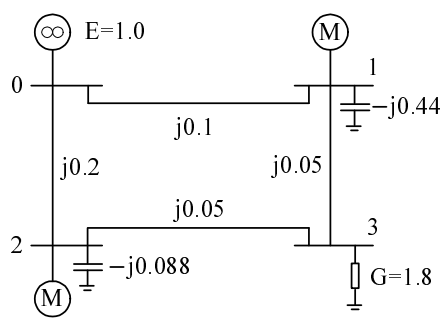

Fig. 16. 4-bus system from [13]

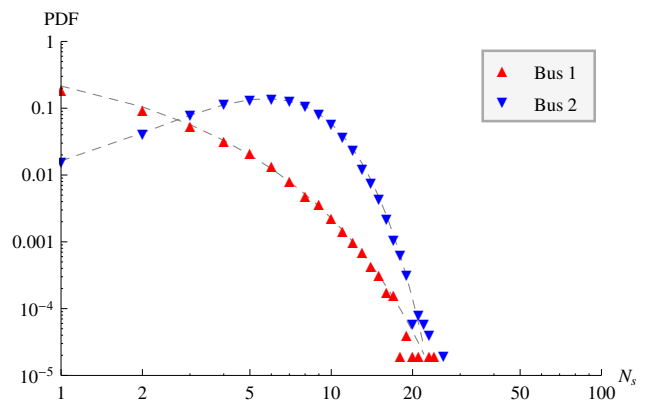

Fig. 17. PDFs of number of motors stalled $N_{s}$ in 4-bus system

buses, until the risk of cascading motor stall at each bus was reduced to acceptable levels.

To give an example of this approach, consider the 4-bus test system ${ }^{2}$ from [13] shown in Fig. 16. Motor loads at bus 1 and 2 comprise $60 \%$ of corresponding bus loads and each bus has 100 motors. Fig. 17 shows the PDFs of number of stalled motors at bus 1 and bus 2. $P_{0}^{1}=0.5695$ and $P_{0}^{2}=$ 0.00266. The fitted dashed curves show that the PDFs of bus 1 or 2 can be fit well by the CASCADE model, and $d_{1}=$ $0.006251, p_{1}=0.004464$ and $d_{2}=0.05570, p_{2}=0.001545$. The probability of very large cascades of more than 30 motors on each bus is negligible, but this is an interesting case because the probability of 1 or 2 motors stalling is much larger at bus 1 , whereas the probability of 3 or more motors stalling is larger at bus 2. Since the larger cascades have more impact, it can be argued that bus 2 is the most vulnerable bus and that we want to first mitigate the humped shape of the PDF at bus 2. The humped shape of the PDF at bus 2 is due to the relatively large value of $d_{2}$; that is, the higher impact of the disturbance on the initial voltage drop at bus 2 . Therefore mitigation should first seek to support the voltage at bus 2 to reduce $d_{2}$, for example by adding capacitative support at bus 2. If this mitigation is cost effective, then further mitigation could be taken after re-evaluating the PDFs to re-evaluate the most vulnerable bus. This sequential bus-by-bus approach is a simple approach that does not address at each mitigation the interactions between the buses. For example, the mitigation at bus 2 that is designed to improve the PDF at bus 2 may also tend to improve the PDF at bus 1 . Future work could consider

\footnotetext{
${ }^{2}$ The system parameters are initial load powers satisfy $P_{L}^{1}=5 P_{L}^{2}=2.47$, initial reactive power of bus 1 and 2 are 0.44 and 0.088 respectively, system disturbance is tripping one circuit of parallel lines between bus 0 and 2. Motor parameters are as previously assumed except that the motors at bus 1 are 5 times larger than that of bus 2, and $V_{\min }^{1}=V_{\min }^{2}=0.5, V_{\max }^{1}=0.90$, $V_{\max }^{2}=0.92$.
} 


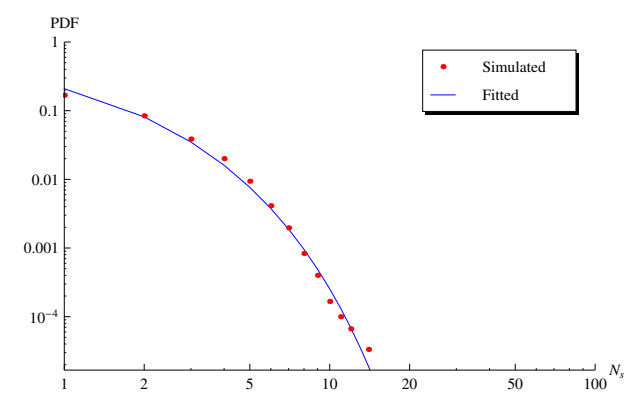

Fig. 18. PDF of number of motors stalled $N_{s}$ with dynamic motor models following a short circuit.

more sophisticated mitigations that simultaneously optimize the PDFs at several buses.

The mitigation approach presented above uses PDFs of number of motors stalled at each bus, but it is straightforward to multiply the PDFs by the value of the motor loads at each bus to obtain the distribution of cascading stall risk at each bus. This enables risk-based consideration of both the probability and impact of the cascades. The thrust of this section is to indicate a plausible path by which the results in this initial paper could be turned into a practical risk-based design procedure to mitigate cascading stall risk.

\section{EXAMPLE CONSIDERING MOTOR DYNAMICS}

To give a brief example of a dynamic case study, we consider a short circuit in the single load infinite bus system where all motors are modeled by a standard third order dynamic model [29] ${ }^{3}$. Fig. 18 shows the PDF of the number of motors stalled $N_{s}$ along with the fitting curve of CASCADE model. The fault clearing time $T_{c l}$ is chosen so that all motors are marginally stable when their $V_{c r}$ are typically sampled. It can be seen that using a dynamic and more detailed motor model does not change the good fit of the PDF with the CASCADE model. The probability of zero motors stalling $P_{0}$ is 0.6721 , which is close to the probability 0.606 estimated in section VI. Obviously, previous studies under quasistatic assumption can be regarded as special cases of general dynamic scenarios, where $T_{c l}$ is set to 0 artificially. Although systematic future work is clearly required, this example suggests that our results with quasistatic assumption can be robust to elaboration with full dynamic process.

Furthermore, with time domain simulation the load bus voltage $V$ at any snapshot can be observed. Fig. 19 compares the PDFs of $N_{s}$ at steady state and $V$ at five snapshots of time, where all PDFs are preprocessed as documented in section V-D. Apparently, except for the PDF of $V$ at $40 \mathrm{~s}$ due to limited samples, the PDFs are essentially similar, indicating

\footnotetext{
${ }^{3}$ The system parameters are same as in section V-A. The short circuit lowers the infinite bus voltage $E$ to 0.1 for a time period of $T_{c l}=0.130 \mathrm{~s}$ and then trips one circuit of double transmission lines. Because the system is subjected to a big disturbance, the motors are very prone to stall. To partially offset this tendency, $V_{c r}$ is assumed to be in the interval $[0.5,0.85]$ for all 100 motors. The time domain dynamic simulation uses trapezoidal integration with time step $0.005 \mathrm{~s}$. To ensure all motors and the system reaching steady state, the dynamic simulation continues until the load bus voltage $V$ changes less than $10^{-6}$ for a continuous $3 \mathrm{~s}$ period.
}

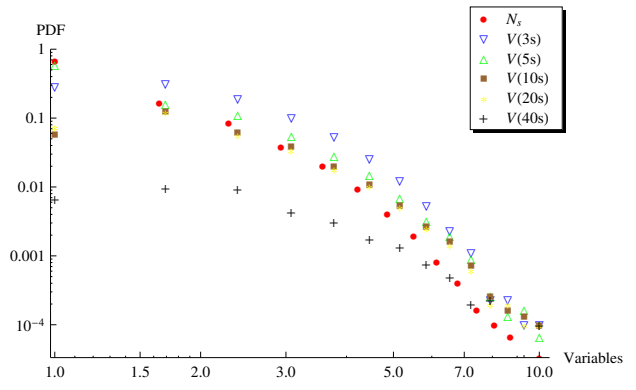

Fig. 19. Distributions of scaled load bus voltage $V$ at 5 snapshots with dynamic motor models following a short circuit.

the existence of a linkage between steady state and dynamic process that is more complex than the linkage among different steady state variables in section V-D.

\section{COMPARISON With COMPOSITE LOAD MODELING}

We briefly compare our approach to modeling cascading motors to an established approach to composite load modeling that is the result of decades of work to reproduce system dynamics in the WECC system [9], [19], [20]. The composite load models in [20] include both static and dynamic models of four types of motor. While we probabilistically quantify the consequences of motor variability and uncertainty, [20] chooses a credible worst case and then proceeds deterministically. Our modeling equivalences the rest of the grid, whereas [20] equivalences all motors of the same type. The equivalenced motors either stall or do not stall, so that the intermediate outcomes of some motors stalling cannot be represented. Table I summarizes the comparison, and it can be seen that the two approaches are complementary and at different stages of development.

\section{COnClusion}

We study the cascading stall and bus voltage decline of 100 motors in a simple one load power system and a 4-bus 2-load small system. The stall is initiated by a power system fault, and some of the motor parameters are random in order to model some of the variation and uncertainty of the motors expected in practice. The probability distribution of the number of motors stalled can be obtained with a quasistatic model of the motor stall. The quasistatic model is orders of magnitude faster than a full dynamic simulation, without losing the accuracy of steady state of cascading stall when assumptions are met. Some very fast insights into the effect of parameters on cascading stall are also possible by running a typical sample of the quasistatic model.

After the onset of possible stall, the probability of no stall decreases exponentially as the initial bus load increases. Near the onset of possible stall, the extent of likely cascading is sensitive to parameters. The distribution of initial motor power has a minor impact on the extent of cascading, and this implies that equal initial motor powers can be assumed without much error. The extent of cascading as determined from distributions of cascading stall number, load bus voltage 
TABLE I

COMPARISON OF APPROACHES

\begin{tabular}{lll} 
& probabilistic cascading stall & composite dynamic load models \\
\hline objective & quantify chances of cascading & reproduce system dynamics \\
analysis type & probabilistic model of load variability & deterministic sample of credible worst case \\
model detail & locally interacting motors & composite loads for entire grid \\
equivalencing & rest of transmission system & all machines of similar type \\
maturity & first analysis on simple system & implemented in industry \\
\hline
\end{tabular}

and load power have similar characteristics. This commonality may offer guidance in future work when looking for patterns in the statistics of observed bus measurements.

Since cascading stall is neither routinely occurring (because of engineering to avoid this) nor non-existent, we expect that the cases of most engineering interest are those in which cascading stall of some of the motors sometimes happens. In these cases, the outcome of the cascading motor stall can be fit with the outcome of the CASCADE model, a high-level and generic probabilistic model of cascading failure. This shows that cascading motor stall has properties common to other cascading processes in engineering and science, and gives another engineering problem to which the CASCADE model can be applied. Moreover, the simplicity of the CASCADE model allows the motor stall to be better understood, and may in future work suggest ways to better quantify the extent of cascading motor stall.

\section{APPENDIX}

The induction motor parameters used in the paper are $r_{1}=0.0456, x_{1}=0.295, r_{2}=0.02, x_{2}=0.12, r_{m}=0.35$, $x_{m}=3.5, \alpha=0.15, p=2.0, T_{J}=2.0 \mathrm{~s}$.

\section{REFERENCES}

[1] C. W. Taylor, Power system voltage stability, McGraw-Hill, NY, 1994.

[2] IEEE/CIGRE Joint Task Force on Stability Terms and Definitions, "Definition and classification of power system stability," IEEE Trans. Power Syst., vol.19, no.2, pp. 1387-1401, May 2004.

[3] T. V. Cutsem, C. Vournas, Voltage stability of electric power systems, Kluwer Academic Publishers, Boston, 1998.

[4] J. A. Diaz de Leon II, C. W. Taylor, "Understanding and solving shortterm voltage stability problems," in Proc. IEEE Power Eng. Soc. Summer Meeting, 2002, vol.2, pp. 745-752.

[5] NERC Transmission issues and System protection and control subcommittees, "Fault-induced delayed voltage recovery," June 2009.

[6] IEEE Task force on load representation for dynamic performance, "Bibliography on load models for power flow and dynamic performance simulation," IEEE Trans. Power Syst., vol.10, no.1, pp.523-538, Feb 1995.

[7] IEEE Task force on load representation for dynamic performance, "Standard load models for power flow and dynamic performance simulation," IEEE Trans. Power Syst., vol.10, no.3, pp. 1302-1313, Aug. 1995.

[8] C. W. Taylor, D. C. Erickson, "Recording and analyzing the July 2 cascading outage," IEEE Computer Application in Power, vol.10, no.1, pp. 26-30, Jan. 1997.

[9] D. N. Kosterev, C. W. Taylor, W. A. Mittelstadt, "Model validation for the August 10, 1996 WSCC system outage," IEEE Trans. Power Syst., vol.14, no.3, pp. 967-974, Aug. 1999.

[10] B. R. Williams, W. R. Schmus, D. C. Dawson, "Transmission voltage recovery delayed by stalled air conditioner compressors," IEEE Trans. Power Syst., vol.7, no.3, pp. 1173-1181, Aug. 1992.

[11] J. W. Shaffer, "Air conditioner response to transmission faults," IEEE Trans. Power Syst., vol.12, no.2, pp. 614-621, May 1997.

[12] Y. Sekine, H. Ohtsuki, "Cascaded voltage collapse," IEEE Trans. Power syst., vol.5, no.1, pp. 250-256, Feb. 1990.
[13] C.D. Vournas, G.A. Manos, "Modeling of stalling motors during voltage stability studies," IEEE Trans. Power syst., vol.13, no.3, pp. 775-781, Aug. 1998.

[14] D. H. Popovic, I. A. Hiskens, D. J. Hill, "Stability analysis of induction motor networks," Electrical Power \& Energy Systems, vol.20, no.7, pp. 475-487, 1998.

[15] L. Pereira, D. Kosterev, P. Mackin, D. Davies, J. Undrill, W. Zhu, "An interim dynamic induction motor model for stability studies in the WSCC," IEEE Trans. Power Syst., vol.17, no.4, pp. 1108-1115, Nov. 2002.

[16] A. M. Stankovic, B. C. Lesieutre, "Parametric variations in dynamic models of induction machine clusters," IEEE Trans. Power Syst., vol.12, no.4, pp. 1549-1554, Nov. 1997.

[17] J. F. Zhang, C. T. Tse, K. W. Wang, C. Y. Chung, "Voltage stability analysis considering the uncertainties of dynamic load parameters," IET Gener. Transm. Distrib., vol.3, no.10, pp. 941-948, 2009.

[18] J. Ma, R. He, D.J. Hill, "Load modeling by finding support vectors of load data from field measurements," IEEE Trans. Power Syst., vol.21, no.2, pp. 726-735, May 2005.

[19] D. Kosterev, A. Meklin, J. Undrill, B. Lesieutre, W. Price, D. Chassin, R. Bravo, S. Yang, "Load modeling in power system studies: WECC progress update," IEEE PES General Meeting, Pittsburgh, PA USA, July 20-24, 2008.

[20] B. Lesieutre, R. Bravo, R. Yinger, D. Chassin, H. Huang, N. Lu, I. Hiskens, and G. Venkataramanan, Load modeling transmission research, Final Report, California Institute for Energy and Environment, March 2010. Online: http://www.uc-ciee.org/downloads/LM_Final_Report.pdf.

[21] IEEE PES CAMS Task Force on Understanding, Prediction, Mitigation and Restoration of Cascading Failures, "Initial review of methods for cascading failure analysis in electric power transmission systems," IEEE PES General Meeting, Pittsburgh, PA USA, July 20-24, 2008.

[22] I. Dobson, B. A. Carreras, V. E. Lynch, D. E. Newman, "Complex systems analysis of series of blackouts: cascading failure, critical points, and self-organization," Chaos, vol.17, 026103, 2007.

[23] I. Dobson, B. A. Carreras, D. E. Newman, "A loading-dependent model of probabilistic cascading failure," Probability in the Engineering and Informational Sciences, vol.19, pp. 15-32, 2005.

[24] H. Ren, I. Dobson, "Using transmission line outage data to estimate cascading failure propagation in an electric power system," IEEE Trans. Circuits \& Systems II, vol. 55, no. 9, Sept. 2008, pp. 927-931.

[25] I. Dobson, J. Kim, K.R. Wierzbicki, "Testing branching process estimators of cascading failure with data from a simulation of transmission line outages," Risk Analysis, vol. 30, no. 4, 2010, pp. 650-662.

[26] I. Dobson, B.A. Carreras, "Number and propagation of line outages in cascading events in electric power transmission systems," 48th Annual Allerton Conference on Communication, Control and Computing, Monticello IL USA, September 2010.

[27] J. Kim, I. Dobson, "Approximating a loading-dependent cascading failure model with a branching process," IEEE Transactions on Reliability, vol. 59, no. 4, December 2010, pp. 691-699.

[28] H. Wu, R. Guo, Z. Han, Y. Cao, "Initial study on macro laws of induction motor cascading stall," Automation of Electric Power Systems, vol.34, no.13, pp. 1-5, 2010. (in Chinese)

[29] X. F. Wang, Y. H. Song, M. Irving, Modern power systems analysis, Springer-Verlag, New York, 2008.

Hao Wu (M'10) received B.A. from Shanghai Jiaotong University, Shanghai, China and Ph.D. from Zhejiang University, Hangzhou, Zhejiang, China. All degrees are in electrical engineering. He joined Zhejiang University in 2002, visited Hong Kong Polytechnic University from June 2003 to September 2004 and University of Wisconsin-Madison from June 2009 to March 2011.

Ian Dobson (F'06) received the Ph.D. in electrical engineering from Cornell University. He is currently Sandbulte professor in electrical and computer engineering at Iowa State University. 